\title{
Traumatic cardiac arrest: unique considerations for the pediatric patient
}

\author{
Chady El Tawil ${ }^{1}\left[\right.$ D $\cdot$ Pierre-Alexandre LeBlanc ${ }^{2} \cdot$ Suzanne Beno ${ }^{2} \cdot$ Joe Nemeth $^{1}$
}

Received: 22 January 2022 / Accepted: 8 February 2022 / Published online: 21 February 2022

(C) The Author(s), under exclusive licence to Canadian Association of Emergency Physicians (CAEP)/ Association Canadienne de Médecine d'Urgence (ACMU) 2022

Keywords Pediatric emergency $\cdot$ Traumatic arrest $\cdot$ Algorithm $\cdot$ Resuscitation

\section{Dear Editor,}

Trauma remains the leading cause of mortality in children and youth globally [1]. Mortality in pediatric trauma follows the trimodal curve described in adults, with relatively more deaths occurring early [2]. Traumatic brain injury, proportionately higher in children, is the predominant etiology of trauma-related mortality, with preventable death being primarily attributed to hemorrhage across all age groups [2]. The most common mechanisms of injury globally leading to pediatric arrest include motor vehicle collisions, followed by firearm-related injuries [1].

Historically, the resuscitation of a patient suffering an out-of-hospital traumatic cardiac arrest was considered futile. With a more physiologically targeted approach to the management of traumatic cardiac arrest, survival rates in adults have in recent years significantly improved. Traumatic and non-traumatic cardiac arrest have different pathophysiological etiologies. While non-traumatic cardiac arrest in adults is most frequently due to primary myocardial dysfunction, patients with traumatic cardiac arrest succumb most commonly to traumatic brain injury, followed by either hypoxia or insufficient preload as a result of intravascular depletion from hemorrhage or obstructive shock via tension pneumothorax or cardiac tamponade.

Life-threatening pediatric trauma is a high acuity lowfrequency event, occurring far less frequently than within

Chady El Tawil

chady.eltawil@mcgill.ca

1 Division of Pediatric Emergency Medicine, Department of Pediatrics, Montreal Children's Hospital, McGill University Health Centre, Montreal, QC, Canada

2 Division of Emergency Medicine, Hospital for Sick Children, University of Toronto, Toronto, ON, Canada adult populations with incidence of 10.4 vs 75.9 per 100.000 in children vs adults, respectively [3]; thus, research driving this care is both more difficult to perform and consequently often less robust. As such, adult-based evidence and guidelines are frequently extrapolated to children when specific pediatric data are lacking.

Recent research, however, is confirming and subsequently promoting that resuscitative methods used in adult patients (e.g., resuscitative thoracotomies) have benefit in all age groups [4]. Such procedures for pediatric patients are currently more frequently undertaken in adult centres compared to pediatric centres [4].

Pediatric traumatic cardiac arrest management is evolving, and clinical algorithms are emerging worldwide [5]. Guidelines in adult trauma are clear and consistent with respect to rapidly addressing reversible etiologies of arrest for achieving survival. While pediatric evidence is less robust and nuances in clinical presentation and etiologies exist, emerging literature supports the implementation of similar if not identical guidelines in children. Further outcome-driven research in children specifically addressing areas of controversy, including "termination of resuscitation" protocols in the field, utility of CPR and epinephrine use, and importantly procedural resuscitative competency, is vital for informing evidence-based pediatric traumatic cardiac arrest protocols with the ultimate goal of improving survival when possible and providing exceptional end-of-life care when not.

Author contribution The four authors contributed to the manuscript by actively searching the literature, drafting, and finalizing the manuscript. CET and PAL drafted the first manuscript, it was revised critically by $\mathrm{JN}$ and SB. All the authors approved the final version and agree to be accountable for all aspects of the work. No other authors participated actively or passively in the article to be acknowledged. 


\section{Declarations}

Conflict of interest The authors declare that they have no conflict of interest and that they did not receive any financial support.

\section{References}

1. Cunningham RM, Walton MA, Carter PM. The major causes of death in children and adolescents in the United States. N Engl J Med. 2018;379(25):2468-75.

2. McLaughlin C, Zagory JA, Fenlon M, Park C, Lane CJ, Meeker D, et al. Timing of mortality in pediatric trauma patients: a national trauma data bank analysis. J Pediatr Surg. 2018;53(2):344-51.

3. Centers for Disease Control and Prevention, National Center for Health Statistics. Underlying Cause of Death 1999-2020 on CDC
WONDER Online Database, released in 2021. Data are from the Multiple Cause of Death Files, 1999-2020, as compiled from data provided by the 57 vital statistics jurisdictions through the Vital Statistics Cooperative Program. Accessed at http://wonder.cdc. gov/ucd-icd10.html. Accessed date 22 Jan 2022

4. Prieto JM, Van Gent JM, Calvo RY, Rooney AS, Martin MJ, Sise MJ, et al. Nationwide analysis of resuscitative thoracotomy in pediatric trauma: time to differentiate from adult guidelines? J Trauma Acute Care Surg. 2020;89(4):686-90.

5. Vassallo J, Nutbeam T, Rickard AC, Lyttle MD, Scholefield B, Maconochie IK, et al. Paediatric traumatic cardiac arrest: the development of an algorithm to guide recognition, management and decisions to terminate resuscitation. Emerg Med J. 2018;35(11):669-74. 